\title{
Combining Model Learning and Data Analysis to Generate Models of Component-based Systems*
}

\author{
Sébastien Salva ${ }^{1}$, Elliott Blot ${ }^{1}$, and Patrice Laurencot ${ }^{1}$ \\ LIMOS CNRS UMR 6158, Clermont Auvergne University, \\ sebastien.salva@uca.fr, eblot@isima.fr, laurencot@isima.fr
}

\begin{abstract}
Finding bugs in systems without model is well-known to be challenging and costly. But, most of today's developers think that writing models is also a hard and error-prone task. In this context, this paper addresses the problem of learning a model, from a componentbased system, which captures and separates the behaviours of components and encodes their synchronisations. We present a passive model learning method called COnfECt to infer such models from execution traces in which no information is provided to identify components. We describe the two main steps of COnfECt in this paper and show some preliminary experimentations on real systems.
\end{abstract}

Keywords: Model learning; Passive learning; Reverse engineering; Component-based systems.

\section{Introduction}

Software testing aims at assessing the quality of the features offered by a system in terms of conformance, security, performance, etc., to discover and correct its defects. Nowadays, testing is essentially performed by means of test cases written by hand, which is often a long, difficult and error-prone task. To make this task easier, model learning approaches have proven to be valuable for recovering models that can be exploited by many software engineering stages, e.g. testing.

Although the generation of behavioural models has been greatly studied, little attention has been given to the learning of models from component-based systems. Yet, most of the systems being currently developed are made up of reusable features or communicating components that interact together. These observations motivate this work, which adresses the challenge of how to learn a model from its traces, in such a way that the model captures the behaviour of every component of the System Under Learning (SUL) and their synchronisations.

For this purpose, we designed the method COnfECt (COrrelate Extract Compose) for learning models of component-based systems. Its main originality is that it does not require any preliminary identification information about components. COnfECt learns a system of LTSs (Labelled Transition Systems) from

* Research supported by the VASOC Project and the French Region Auvergne-RhôneAlpes (https://vasoc.limos.fr/) 
traces (passive learning), which captures the behaviours of every component by a LTS and shows how they are synchronised together. COnfECt is composed of two main steps called Trace Analysis 85 Extraction and LTS synchronisation which are going to be developped in this paper.

Paper organisation: Section 2 introduces the two steps of the COnfECt approach. The next section summarises the results of a preliminary evaluation on an IOT (Internet Of Things) device. Finally we conclude in Section 4.

\section{The COnfECt Approach}

Beforehand, we recall that the LTS model, we use in this paper, is defined in terms of states and transitions labelled by actions, taken from a general action set $\mathcal{L}$, which expresses what happens (a more complete definition can be found in $[2])$. We also define special actions of the form call $_{-} C_{i}$ and return $C_{i}$ to model component calls with $C_{i}$ referring to a LTS. Actions of the form call_ $C_{i}$ and return_ $C_{i}$ synchronise pairs of LTSs as described in[1]. The execution of $C_{i}$ starts with the label call_ $C_{i}$ and ends when the transition return $C_{i}$ is fired.

\subsection{Overview of COnfECt}

The COnfECt method aims to infer a system of LTSs $S C$ from the traces of $S U L$, in such a way that $S C$ captures the behaviours of the $S U L$ components and their synchronisations. COnfECt initially requires the set of traces of $S U L$, denoted Traces $(S U L)$, to analyse the system behaviours and identify components. We suppose that each component can be identified by its behaviour, materialised by action sequences. And the more traces, the more correct the component detection will be. $S U L$ can be indeterministic, uncontrollable or can have cycles among its internal states. However, we assume $S U L$ and Traces $(S U L)$ obey certain restrictions. We consider that $S U L$ has components whose observable behaviours are not carried out in parallel. One component is executed at a time from its initial state to one of its final states. Furthermore, we consider that traces are collected in a synchronous manner (by means of synchronous communications) to avoid the interleaving of actions. Traces can be collected by means of monitoring tools or extracted from log files. We assume that $\operatorname{Traces}(S U L)$ does not include actions expressing the calls of components.

Furthermore, although this task is costly and important, we do not focus this work on the trace formatting, hence, we assume having a mapper, which is a tool often required in model learning to transform raw execution traces into higher level representations.

COnfECt has two main successive steps illustrated in Figure 1. The first step, called Trace Analysis \& Extraction tries to detect components in Traces(SUL), which is partitioned into a set of trace sets called STraces. Each trace set of STraces captures some behaviours of one component. The second step, called LTS Synchronisation, takes the set STraces and starts with the generation of one LTS for each trace set of STraces. This step also proposes different synchronisation strategies to generate a system of LTSs $S C$, before merging equivalent states with kTail. 


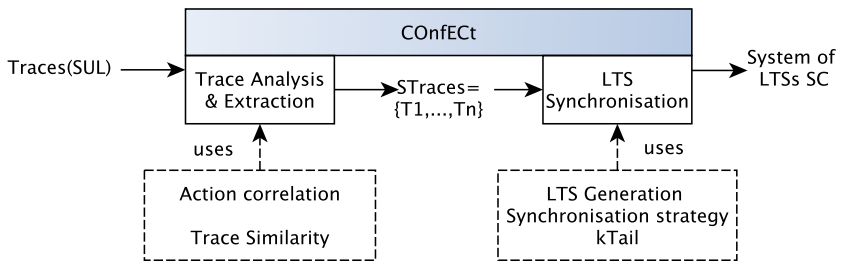

Fig. 1: The COnfECt approach overview

\subsection{Trace Analysis \& Extraction}

The aim of this step is to identify the different components in the traces of Traces $(S U L)$. The algorithm, which is given in [2] is divided into three procedures. The first one, Inspect analyses the traces and segments them in subsequences. We define a Correlation Coefficient to evaluate the correlation of successive actions in Traces $(S U L)$, i.e. the degree to which successive actions are associated with regard to Traces $(S U L)$. We define the Correlation coefficient between two actions by means of a utility function, which involves a weighting process for representing user priorities and preferences. We have chosen the technique Simple Additive Weighting (SAW) [3], which allows the interpretation of these preferences with weights. This factor must take a value between 0 and 1 , and needs to be appraised, depending of the context.

From this Correlation coefficient, we define a relation to express the notion of strong correlation. We say that strong-corr $\left(\sigma_{1}\right)$ holds when $\sigma_{1}$ has successive actions that strongly correlate. Besides, we compare two sequences with the relation $\sigma_{1}$ mismatch $\sigma_{2}$, which holds when the last event of $\sigma_{1}$ does not correlate strongly with the first one of $\sigma_{2}$.

The second procedure Extract whose algorithm is also given in [2] creates recursively different sequences to express component calls. It takes every trace $\sigma$, transforms it and stores the new trace into a set $T_{j}$, by the means of the coefficient correlation.

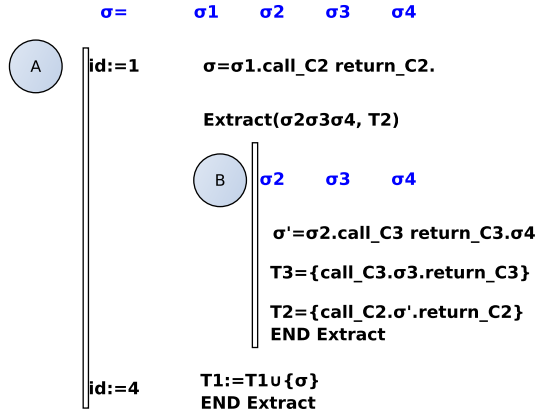

(a) Procedure Extract steps

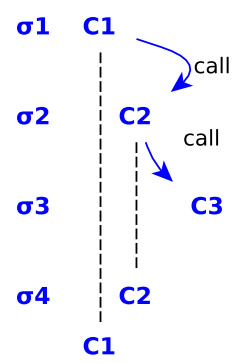

(b) Component call

Fig. 2: Sequence extraction example

Example 1. Let us illustrate the procedure Extract with the example of Figure $2 \mathrm{a}$. The procedure takes as input a trace initially segmented into 4 sub-sequences 
by the correlation coefficient. A) We start at $\sigma_{1}$ and suppose that no other subsequence is strongly correlated with $\sigma_{1}$. The sequence $\sigma_{2} \sigma_{3} \sigma_{4}$ is hence extracted and replaced by the actions call_C2 return_C2, which model the call of a component $C 2$. The procedure is recursively called with $\operatorname{Extract}\left(\sigma^{\prime}=\sigma_{2} \sigma_{3} \sigma_{4}, T_{2}\right)$. B) We now suppose $\sigma_{2} \sigma_{4}$ strongly correlate, thus $\sigma_{3}$ is extracted and the sequence $\sigma^{\prime}$ becomes $\sigma^{\prime}=\sigma_{2}$.call_C $C 3$ return $C$ C $3 . \sigma_{4}$. The extracted sequence $\sigma_{3}$ cannot be segmented. It is surrounded with the actions call_C 3 and return_C3 to prepare the LTS synchronisation and to express that C3 is called by another component. The resulting sequence is added to the set $T_{3}$. As $\sigma^{\prime}$ is completely covered, $\sigma^{\prime}$ is surrounded with the actions call_C2 and return_C $C 2$ and added to the new trace set $T_{2}$. At the end of this process, we have recovered the hierarchical component call depicted in Figure 2b and we get three trace sets.

The set $T_{1}$, which holds the modified traces of the initial traces set Traces $(S U L)$, may include traces resulting from several components. We call the third procedure Separate for trying to partition T1, to build the set STraces such that a trace set $T$ of $S$ Traces is produced by one component. For that, we evaluate the trace similarity with regard to the actions shared between pairs of traces. Among the different available coefficients, we chose the Overlap coefficient because the action sets used by two traces may have different sizes. Then a clustering technique is used to get the equivalence classes. The procedure Separate is implemented with a Similarity threshold here.

\subsection{LTS Synchronisation}

The previous step of COnfECt has segmented, extracted and modified the traces of Traces $(S U L)$ in such a way that each traces set contains the behaviour of only one component. We generate a LTS from every traces set, where each trace represent a path of a tree-like LTS. These LTSs include actions of the form call_ $C_{i}$ and return $C_{i}$. These actions were added in the previous step to prepare the synchronisation of components with LTSs. We proposes different synchronisation strategies, which provide systems of LTSs with different levels of generalisation. The strict synchronisation limits over-generalisation, and used only kTail to merge equivalent states. The weak synchronisation aims at reducing the number of models and allows repetitive components calls, its uses a LTS similary coefficient to merge models by means of a clustering technique. The strong synchronisation generate callable-complete LTSs, i.e., the LTS can call any other LTS of the system from any states.

Example 2. Let us illustrate this step with the set STraces of Figure 3. The traces $T 1$ to $T 4$ are obtained from the step Trace Analysis \& Extraction on a trace collected from a real smart thermostat device at the HTTP level. This trace, composed of 16 actions, was formatted to keep the Urls and some data, e.g., the temperature.

We choose to apply the Weak synchronization strategy. A similarity matrix is computed by means of the LTS Similarity coefficient. Figure 4a shows the matrix obtained with the four LTSs of our example. We can observe that two 


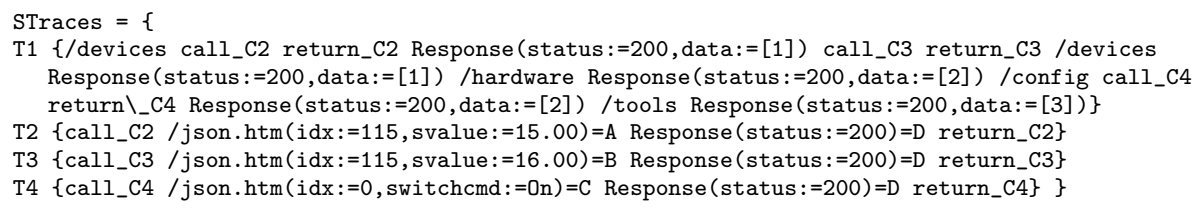

Fig. 3: Example of formatted trace segmented into 4 trace sets.

classes of similar LTSs emerge in this matrix: $\left(C_{1}\right)$ and $\left(C_{2}, C_{3}, C_{4}\right)$. A clustering technique is used to generate these classes. The LTSs of each cluster are then joined by means of a disjoint union.

\begin{tabular}{c|c|c|c|c} 
& $C_{1}$ & $C_{2}$ & $C_{3}$ & $C_{4}$ \\
\hline$C_{1}$ & 1 & 0,38 & 0,38 & 0,38 \\
\hline$C_{2}$ & 0,38 & 1 & 1 & 0,8 \\
\hline$C_{3}$ & 0,38 & 1 & 1 & 0,8 \\
\hline$C_{4}$ & 0,38 & 0,8 & 0,8 & 1
\end{tabular}

(a) LTS Similarity matrix

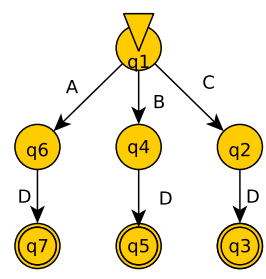

(b) LTS C_2_3_4 mod-(c) Reduced elling the sensors LTS C_2_3_4

Fig. 4: LTS results

From the trace sets of Figure 3, we obtain the two LTS clusters: $\left(C_{1}\right)$ and $\left(C_{2}, C_{3}, C_{4}\right)$, the first one expressing the behaviour of the Web interface, and the other one, the component that sends data. Figure $4 \mathrm{~b}$ depicts the LTS $C_{234}$ derived from the second cluster. The LTS $C_{234}$ holds two equivalent state classes $(q 2, q 4, q 6)$ and $(q 3, q 5, q 7)$. kTail merges them and returns the LTS of Figure 4c.

\section{Preliminary Evaluation}

We have implemented COnfECt in a prototype tool on which we conducted several experiments. We initially collected traces from an IOT device, a smart connected thermostat. It integrates 3 components providing HTTP traces. Several experiences have been performed, only five of them are provided in Table 1 and 2 : exp 1 and 2, traces of only one component is recovered, exp 3 and 4 , traces of 2 components, and the last one with all the components.

Firstly, we evaluated the capability of COnfECt to recover the correct number of components, and then we compared the number of states and transitions with kTail. The tool, the trace sets and results are available here ${ }^{1}$. In Table 1 , for exp. 1 to 5, the number of LTSs is equal to the number of real components with the Weak and Strong strategies, but not with the Strict strategy. This strategy segments traces, which are lifted to the level of LTS, but these are not merged.

Table 2 gives the number of states and transitions of all the LTSs generated by COnfECt in Exp. 1 to 5. We also provide the number of states and transitions of these LTSs after removing the transitions labelled by the synchronisation actions in the last three columns. For comparison purposes, we applied kTail

\footnotetext{
${ }^{1}$ https://github.com/Elblot/COnfECt
} 


\begin{tabular}{|c|c|c|c|c|}
\hline Exp & \# real Components & Strict & Weak & Strong \\
\hline Exp. 1 & 1 & 10 & 1 & 1 \\
\hline Exp. 2 & 1 & 1 & 1 & 1 \\
\hline Exp. 3 & 2 & 85 & 2 & 2 \\
\hline Exp. 4 & 2 & 67 & 2 & 2 \\
\hline Exp. 5 & 3 & 173 & 3 & 3 \\
\hline
\end{tabular}

Table 1: Number of components detected by COnfECt.

\begin{tabular}{|c|c|c|c|c|c|c|c|c|c|c|c|c|c|c|}
\hline \multirow{2}{*}{ Exp. } & \multicolumn{2}{|c|}{ kTail } & \multicolumn{2}{|c|}{ Strict } & \multicolumn{2}{|c|}{ Weak } & \multicolumn{2}{|c|}{ Strong } & \multicolumn{2}{|c|}{ Strict+hide } & \multicolumn{2}{|c|}{ Weak+hide } & \multicolumn{2}{|c|}{ Strong+hide } \\
\hline & \#states & \#trans & \#states & \#trans & \#states & \#trans & \#states & \#trans & \#states & \#trans & \#states & \#trans & \#states & \#trans \\
\hline Exp 1 & 40 & 66 & 152 & 169 & 46 & 78 & 60 & 150 & 120 & 137 & 39 & 70 & 36 & 67 \\
\hline Exp 2 & 6 & 8 & 6 & 8 & 6 & 8 & 6 & 8 & 6 & 8 & 6 & 8 & 6 & 8 \\
\hline Exp 3 & 60 & 115 & 731 & 691 & 104 & 188 & 72 & 183 & 399 & 359 & 71 & 124 & 36 & 85 \\
\hline Exp 4 & 22 & 47 & 496 & 470 & 41 & 81 & 25 & 57 & 236 & 210 & 24 & 55 & 10 & 23 \\
\hline Exp 5 & 85 & 175 & 1307 & 1185 & 158 & 286 & 82 & 197 & 627 & 505 & 96 & 169 & 36 & 87 \\
\hline
\end{tabular}

Table 2: Size of the LTSs obtained with kTail and the three strategies of COnfECt. The label "hide" refers to the removal of the LTS transitions labelled by synchronisation actions.

on the same trace sets. As expected, we obtain bigger LTSs with COnfECt than the ones achieved by kTail (excepted with Exp. 2 since there is no trace segmentation). This result comes from the functioning of our method since the LTSs are completed with transitions labelled by synchronisation actions.

The transitions labelled by synchronisation actions help interpret the components combination and are required to compose LTSs, but are not relevant if one want to focus on the component behaviours only. If we remove them, the models achieved by COnfECt become more concise than those obtained with kTail.

\section{Conclusion}

We have introduced COnfECt, a passive model learning method that generates systems of LTSs from execution traces. A system of LTSs captures the behaviours of components and their synchronisations. COnfECt detects component behaviours by analysing traces with a Correlation coefficient and Similarity coefficients. It proposes different LTS synchronisation strategies, which help manage the model generalisation. With this hierarchic component organisation, we believe it offers better readability and comprehensibility than classical learned models, and consequently can be easily used for testing. In future work, we plan to perform more evaluations of COnfECt on several kinds of systems. We also plan to use the models for security testing.

\section{References}

1. van der Bijl, M., Rensink, A., Tretmans, J.: Compositional testing with ioco. In: Petrenko, A., Ulrich, A. (eds.) Formal Approaches to Software Testing. pp. 86-100. Springer Berlin Heidelberg, Berlin, Heidelberg (2004)

2. Salva, S., Blot, E., Laurençot, P.: Combining Model Learning and Data Analysis to Generate Models of Component-based Systems. Limos research report (May 2018), http://sebastien.salva.free.fr/RR-18-05.pdf

3. Yoon, K.P., Hwang, C.L.: Multiple attribute decision making: An introduction (quantitative applications in the social sciences) (1995) 